\title{
A New Fuzzy Bandwidth Carrier Recovery System in GPS for Robust Phase Tracking
}

\author{
Wei-Lung Mao, Hen-Wai Tsao, Member, IEEE, and Fan-Ren Chang, Member, IEEE
}

\begin{abstract}
Fast carrier recovery loops are essential in high-dynamic global positioning system receivers. A new digital phase-locked loop (DPLL) system incorporating a fuzzy bandwidth controller (FBC) is presented in this letter. The FBC provides a time-varying bandwidth and adjusts the loop filter coefficients to improve DPLL tracking capability. When the phase error or phase difference error becomes large in the presence of significant accelerations, the loop bandwidth increases adaptively and performs rapid locking. Simulation results show that our receiver does achieve a superior performance over conventional tracking loops in terms of shorter settling time and wider frequency ramp range, while preventing the occurrence of cycle slipping.
\end{abstract}

Index Terms-Carrier phase tracking, cycle slipping, digital phase-locked loop (DPLL), fuzzy bandwidth controller (FBC), global positioning system (GPS) receiver.

\section{INTRODUCTION}

C ARRIER PHASE observable is widely used in global positioning systems (GPS) to obtain a centimeter-level positioning accuracy with applications of aircraft precision approaches, missile navigation, and etc. Unfortunately, high dynamics and shadowing may lead to cycle slipping that seriously degrades the ranging accuracy and requires a time-consuming ambiguity search algorithm for rinitialization. Previously, a fuzzy estimation filter [1] has been proposed for missile navigation. However, the model needed an a priori known specific trajectory to train the fuzzy phase-locked loop (PLL) and thus was unsuitable for practical receiver. Lee et al. [7] presented an adaptive PLL capable of varying the loop bandwidth by increasing the amount of charge-pump current. An alternative scheme operating by the control on the reference frequency and frequency division ratio was proposed for frequency synthesizer [8]. Thus, these adaptive bandwidth methods are only applicable for analog PLL architectures. In carrier loop design, a wider bandwidth is needed for rapid acquisition, whereas a smaller bandwidth is preferred for noise performance [2]. To enhance both carrier tracking and phase estimation accuracy simultaneously in GPS receivers, the digital bandwidth-adjustment scheme is indispensable to overcome this difficulty.

In this letter, we propose a new carrier recovery system composed of a third-order digital phase-locked loop (DPLL) and a fuzzy bandwidth controller (FBC) to achieve robust carrier

Manuscript received May 26, 2003; revised August 5, 2003. The associate editor coordinating the review of this manuscript and approving it for publication was Prof. Visa Koivunen.

The authors are with the Integrated System Laboratory, Department of Electrical Engineering, Graduate Institute of Electronics Engineering, National Taiwan University, Taipei, Taiwan, R.O.C. (e-mail: wlmao@ccsun.mit.edu.tw; tsaohw@cc.ee.ntu.edu.tw; frchang@cc.ee.ntu.edu.tw).

Digital Object Identifier 10.1109/LSP.2004.824020 phase tracking. The FBC provides a means of converting a control strategy comprised of a set of linguistic rules into a bandwidth-control operation and requires only moderate computational complexity. Under realistic mobile conditions, the carrier phase signal is modeled as a sum of three components, these being 1) phase offset, 2) frequency offset, and 3) frequency ramp offset. When the phase error or phase difference error increases due to large dynamics, the FBC provides an adaptive bandwidth to the DPLL and results in fast acquisition. If both absolute phase and phase difference errors are small, the loop bandwidth will reduce and thus improves the positioning accuracy. This new adaptive-bandwidth method has been shown to shorten the pull-in process and widen the locking range in comparison with conventional loops under a variety of dynamic environments.

\section{Mobile CARrier PHASE Model}

A detailed block diagram of the proposed GPS carrier loop is illustrated in Fig. 1(a). The received sample observation can be represented as

$$
r_{i}=\sqrt{2 P} C\left[i T_{s}-\tau\right] \cos \left(2 \pi f_{\mathrm{IF}} i T_{s}+\theta_{i}\right)+n_{i}
$$

where $P$ is the average power of the received signal, $C[\bullet]$ is the coarse/acquisition (C/A) code sequence, $T_{s}$ is the sample period, $\tau$ is the code transmission delay time, $\omega_{\mathrm{IF}}$ is the receiver intermediate radian frequency, and $\theta_{i}$ is the unknown carrier phase to be estimated. The noise component $n_{i}$ is modeled as sample of a zero-mean white Gaussian noise with two-sided power spectral density of $N_{0} / 2 \mathrm{~W} / \mathrm{Hz}$, and the jamming source is not considered in this letter. The mathematical model for the carrier phase $\theta_{i}$ can be represented as

$$
\theta_{i}=\theta_{0}+2 \pi f_{0}\left(i T_{s}\right)+\pi \lambda_{0}\left(i T_{s}\right)^{2}
$$

where $\theta_{0}$ (radians) is a constant phase offset, $f_{0}$ (hertz) is the amount of frequency offset due to the Doppler effect, and $\lambda_{0}$ (hertz per second) is the frequency ramp offset of a high-dynamic user. Since the frequency ramp caused by the satellite motion is usually small, we may consider only the motion of vehicles [4]. It is assumed that the user has an acceleration of $a_{c}\left(\mathrm{~m} / \mathrm{s}^{2}\right)$, and the equivalent Doppler frequency ramp becomes

$$
\lambda_{0}=\frac{a_{c} f_{c}}{c} \quad(\mathrm{~Hz} / \mathrm{s})
$$

where $c$ is the speed of light and $f_{c}$ is the GPS L1 carrier frequency $(=1575.42 \mathrm{MHz})$. For a highly dynamic condition with an acceleration of $10 G(G=$ gravitational acceleration with a value of $9.8 \mathrm{~m} / \mathrm{s}^{2}$ ), the frequency ramp $\lambda_{0}$ will be $515 \mathrm{~Hz} / \mathrm{s}$. 


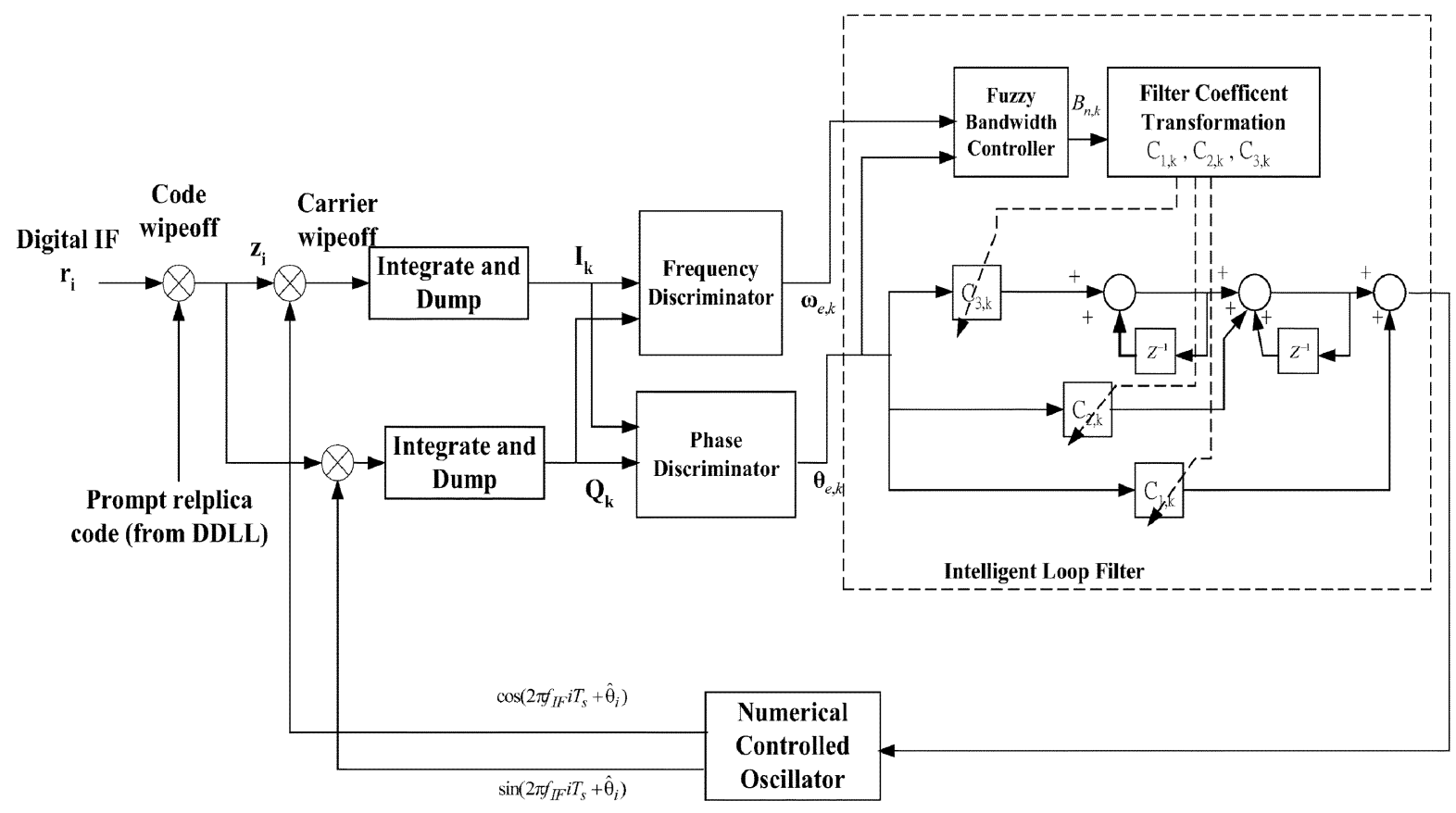

(a)
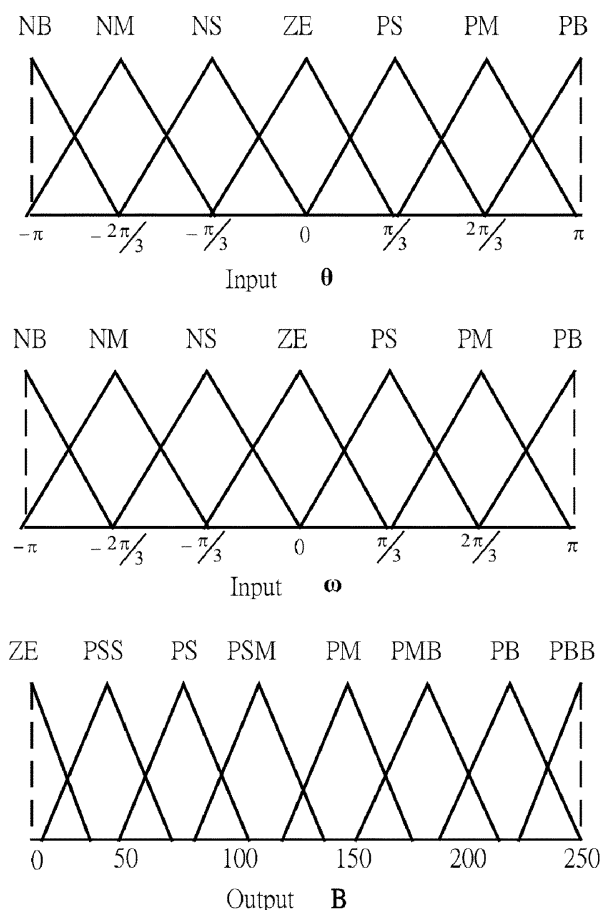

\begin{tabular}{|c|c|c|c|c|c|c|c|}
\hline$\theta$ & $\mathrm{PB}$ & $\mathrm{PM}$ & $\mathrm{PS}$ & $\mathrm{ZE}$ & $\mathrm{NS}$ & $\mathrm{NM}$ & $\mathrm{NB}$ \\
\hline $\mathrm{PB}$ & $\mathrm{PBB}$ & $\mathrm{PB}$ & $\mathrm{PB}$ & $\mathrm{PMB}$ & $\mathrm{PB}$ & $\mathrm{PB}$ & $\mathrm{PBB}$ \\
\hline $\mathrm{PM}$ & $\mathrm{PB}$ & $\mathrm{PMB}$ & $\mathrm{PM}$ & $\mathrm{PSM}$ & $\mathrm{PM}$ & $\mathrm{PMB}$ & $\mathrm{PB}$ \\
\hline $\mathrm{PS}$ & $\mathrm{PMB}$ & $\mathrm{PM}$ & $\mathrm{PS}$ & $\mathrm{PSS}$ & $\mathrm{PS}$ & $\mathrm{PM}$ & $\mathrm{PMB}$ \\
\hline $\mathrm{ZE}$ & $\mathrm{PM}$ & $\mathrm{PS}$ & $\mathrm{PSS}$ & $\mathrm{ZE}$ & $\mathrm{PSS}$ & $\mathrm{PS}$ & $\mathrm{PM}$ \\
\hline $\mathrm{NS}$ & $\mathrm{PMB}$ & $\mathrm{PM}$ & $\mathrm{PS}$ & $\mathrm{PSS}$ & $\mathrm{PS}$ & $\mathrm{PM}$ & $\mathrm{PMB}$ \\
\hline $\mathrm{NM}$ & $\mathrm{PB}$ & $\mathrm{PMB}$ & $\mathrm{PM}$ & $\mathrm{PSM}$ & $\mathrm{PM}$ & $\mathrm{PMB}$ & $\mathrm{PB}$ \\
\hline $\mathrm{NB}$ & $\mathrm{PBB}$ & $\mathrm{PB}$ & $\mathrm{PB}$ & $\mathrm{PMB}$ & $\mathrm{PB}$ & $\mathrm{PB}$ & $\mathrm{PBB}$ \\
\hline
\end{tabular}

(b)

Fig. 1. (a) Proposed fuzzy bandwidth carrier tracking loop. (b) Membership functions and rule table.

\section{NEW FUZZY BANDWIDTH CARRIER TRACKING LOOP}

\section{A. Fuzzy Bandwidth Controller (FBC)}

The integration and dump filters sum up their input data in I- and Q-arms, and the outputs of the digital accumulators [6] become

$$
\begin{aligned}
I_{k}= & \sqrt{2 P} M \sin c\left(\frac{\Delta \omega T}{2}\right) R(\tau-\hat{\tau}) \\
& \cdot \cos [\theta(k T)-\hat{\theta}(k T)]+n_{I, k}
\end{aligned}
$$

$$
\begin{aligned}
Q_{k}= & \sqrt{2 P} M \sin c\left(\frac{\Delta \omega T}{2}\right) R(\tau-\hat{\tau}) \\
& \cdot \sin [\theta(k T)-\hat{\theta}(k T)]+n_{Q, k}
\end{aligned}
$$

where $R(\bullet)$ is the autocorrelation function of C/A codes, $T$ is the loop update time, $\Delta \omega=[\theta(k T)-\theta(k T-T)] / T$, and $M=$ $T / T_{s}$ is the number of samples summed together to update the loop. $\theta(k T)$ and $\hat{\theta}(k T)$ are the phases of the received signal and local carrier signal. $n_{I, k}$ and $n_{Q, k}$ are I- and Q-phase noise components due to noise $n_{i}$. The phase error $\theta_{e, k}$ and phase 
TABLE I

SETTLING TIMES For EACH DPLL SCHEME IN DOPPLER FREQUENCY RAMP ENVIRONMENTS

\begin{tabular}{c|c|c|c|c|c|c|c|c|c|c}
\hline Gravitational acceleration (G) & 1 & 2 & 3 & 4 & 5 & 6 & 7 & 8 & 9 & 10 \\
\hline Frequency ramp offset (Hz/s) & 51.5 & 102.9 & 154.4 & 205.9 & 257.3 & 308.8 & 360.2 & 411.7 & 463.2 & 515 \\
\hline Types of digital carrier loop & \multicolumn{7}{|c}{ Settling time (ms) } \\
\hline Fuzzy bandwidth carrier loop & 18 & 18 & 19 & 20 & 20 & 22 & 23 & 25 & 26 & 28 \\
\hline Conventional 2 2d - order loop [3] & 228 & 230 & 233 & LL & LL & LL & LL & LL & LL & LL \\
\hline Conventional 3 3d - order loop [3] & 715 & 721 & 725 & 982 & LL & LL & LL & LL & LL & LL \\
\hline Conventional 4 $4^{\text {th }}$-order loop [3] & 4341 & 4344 & LL & LL & LL & LL & LL & LL & LL & LL \\
\hline
\end{tabular}

difference error $\omega_{e, k}$ are chosen as input variables of the FBC and given by

$$
\begin{aligned}
& \theta_{e, k}=\arctan \left[\frac{Q_{k}}{I_{k}}\right] \\
& \omega_{e, k}=\arctan \left[\frac{\left(I_{k-1} Q_{k}-I_{k} Q_{k-1}\right)}{\left(I_{k-1} I_{k}-Q_{k} Q_{k-1}\right)}\right] .
\end{aligned}
$$

The output variable of the $\mathrm{FBC}, B_{n, k}$, is the desired loop bandwidth to vary the loop filter parameters accordingly. We choose triangular-shaped membership functions (MFs) for the input and output variables [shown in Fig. 1(b)]. The inference mechanism based on the Mamdani algorithm [5] is utilized. These control rules have the general form

$$
\text { IF } \theta_{e} \text { is } S_{1}^{i} \text { AND } \omega_{e} \text { is } S_{2}^{i}, \quad \text { THEN } B \text { is } S_{3}^{i}
$$

where $\theta_{e}, \omega_{e}$, and $B$ are the linguistic variables, and $S_{1}^{i}, S_{2}^{i}$ and $S_{3}^{i}$ are the corresponding linguistic labels characterized by MFs. The fuzzy output $\bar{B}$ can be determined using the center-average defuzzification [5] formulated as follows:

$$
\bar{B}=\frac{\sum_{i=1}^{n} B_{i} u\left(B_{i}\right)}{\sum_{i=1}^{n} u\left(B_{i}\right)}
$$

where $n$ is the number of fuzzy output sets, $B_{i}$ is the numerical value of $i$ output MF, and $u\left(B_{i}\right)$ represents its membership value at the $i$ th quantization level.

\section{B. Filter Coefficient Transformation}

The closed-loop transfer function of a third-order PLL [3] can be represented as

$$
H(s)=\frac{b \omega_{n} s^{2}+a \omega_{n}^{2} s+\omega_{n}^{3}}{s^{3}+b \omega_{n} s^{2}+a \omega_{n}^{2} s+\omega_{n}^{3}} .
$$

The single-sided noise bandwidth $B_{n}$ for this loop is

$$
B_{n}=\int_{0}^{\infty}|H(j 2 \pi f)|^{2} d f=M \omega_{n}(\mathrm{~Hz})
$$

where $M=\left(a b^{2}+a^{2}-b\right) /(4 a b-4)$ is the ratio of the natural frequency $\omega_{n}$ to the loop bandwidth $B_{n}$. Since most GPS carrier loops are implemented as a digital structure, the bilinear transformation is used to map the continuous-time system into a discrete-time one. We consider the second-order loop filter with a transfer function

$$
L(z)=C_{1}+\frac{C_{2}}{1-z^{-1}}+\frac{C_{3}}{\left(1-z^{-1}\right)^{2}}
$$

where $C_{1}, C_{2}$, and $C_{3}$ are the digital filter coefficients. Inserting (10) into the carrier loop system, the discrete-time transfer function becomes as shown in (11) at the bottom of page, where $C_{d}$ is the gain of phase discriminator, and $C_{n}$ is the gain of the numerically controlled oscillator. The loop filter parameters in terms of loop bandwidth $B_{n}$ can be derived as

$$
\begin{aligned}
C_{1} & =\frac{8 b \omega_{n} T+2 \omega_{n}^{3} T^{3}}{C_{d} C_{n}\left[8+4 b \omega_{n} T+2 a \omega_{n}^{2} T^{2}+\omega_{n}^{3} T^{3}\right]} \\
& =\frac{8 b M^{-1} B_{n} T+2 M^{-3} B_{n}^{3} T^{3}}{C_{d} C_{n}\left[8+4 b M^{-1} B_{n} T+2 a M^{-2} B_{n}^{2} T^{2}+M^{-3} B_{n}^{3} T^{3}\right]} \\
C_{2} & =\frac{8 a \omega_{n}^{2} T^{2}-4 \omega_{n}^{3} T^{3}}{C_{d} C_{n}\left[8+4 b \omega_{n} T+2 a \omega_{n}^{2} T^{2}+\omega_{n}^{3} T^{3}\right]} \\
& =\frac{8 a \omega_{n}^{2} T^{2}-4 \omega_{n}^{3} T^{3}}{C_{d} C_{n}\left[8+4 b M^{-1} B_{n} T+2 a M^{-2} B_{n}^{2} T^{2}+M^{-3} B_{n}^{3} T^{3}\right]}
\end{aligned}
$$

$$
\begin{aligned}
C_{3} & =\frac{8 \omega_{n}^{3} T^{3}}{C_{d} C_{n}\left[8+4 b \omega_{n} T+2 a \omega_{n}^{2} T^{2}+\omega_{n}^{3} T^{3}\right]} \\
& =\frac{8 M^{-3} B_{n}^{3} T^{3}}{C_{d} C_{n}\left[8+4 b M^{-1} B_{n} T+2 a M^{-2} B_{n}^{2} T^{2}+M^{-3} B_{n}^{3} T^{3}\right]}
\end{aligned}
$$

$$
H(z)=\frac{C_{d} C_{n} z^{-1}\left[\left(C_{1}+C_{2}+C_{3}\right)-\left(2 C_{1}+C_{2}\right) z^{-1}+C_{1} z^{-2}\right]}{1+\left[C_{d} C_{n}\left(C_{1}+C_{2}+C_{3}\right)-3\right] z^{-1}+\left[3-C_{d} C_{n}\left(2 C_{1}+C_{2}\right)\right] z^{-2}+\left[C_{d} C_{n} C_{1}-1\right] z^{-3}}
$$




\section{Simulation Results}

Computer simulations have been carried out to evaluate the performance of proposed scheme applied in high-dynamic receivers. The relevant parameters are: the IF carrier $f_{\mathrm{IF}}=1.25 \mathrm{MHz}$, IF sampling frequency $f_{s}=5 \mathrm{MHz}$, and loop update time $T=1 \mathrm{~ms}$. The value of the third-order filter coefficients $a$ and $b$ are chosen as 1.1 and 2.4 [3], respectively. The new fuzzy bandwidth DPLL is compared to the conventional second-, third-, and fourth-order loops with $B_{n}=10 \mathrm{~Hz}$ [3]. The desired dynamic limitations are set as a lock range of $100 \mathrm{~Hz}$ and a frequency ramp range of $515 \mathrm{~Hz} / \mathrm{s}$. The complete rule table for the FBC is shown in Fig. 1(b). Seven linguistic terms are used for each input variables, and 49 rules are developed in this controller. The dynamic tests are conducted at a lowest value of CNR $35 \mathrm{~dB}-\mathrm{Hz}$ [2], and each result is obtained through 2000 independent Monte Carlo simulations.

Table I compares the simulated performances of our fuzzy loop with those of the conventional methods under the acceleration conditions. The constant-bandwidth loops can only operate in smaller frequency ramp ranges (near $220 \mathrm{~Hz} / \mathrm{s}$ ). On the other hand, the FBC provides an adaptive loop bandwidth to the DPLL, and the corresponding tracking capability can be significantly extended to $515 \mathrm{~Hz} / \mathrm{s}$ with a shorter settling time of 28 ms (5\% frequency ramp error specification).

Table II shows the acquisition limitation comparisons between each DPLL scheme. If no fuzzy algorithm is used, loss of locking will occur in conventional loops when the frequency offset is larger than $30 \mathrm{~Hz}$. It is observed that the fuzzy bandwidth scheme can work very well to achieve wider lock range and pull-in range. The significant improvement by using our DPLL is that a robust transient behavior can be performed in kinematic environments.

\section{CONCLUSION}

This letter has presented a new architecture of an intelligent GPS receiver suitable for use under various dynamic conditions. Based on the bandwidth-adjustment criterion, the proposed car-
TABLE II

ACQUISITION LIMITATIONS FOR EACH DPLL SCHEME

\begin{tabular}{c|c|c|c}
\hline $\begin{array}{c}\text { Types of digital } \\
\text { carrier loop }\end{array}$ & Lock range (Hz) & Pull-in range (Hz) & Freq. ramp range (Hz/s) \\
\hline $\begin{array}{c}\text { Fuzzy bandwidth } \\
\text { carrier loop }\end{array}$ & $100.2 \mathrm{~Hz}$ & $134.6 \mathrm{~Hz}$ & $515 \mathrm{~Hz} / \mathrm{s}(10 \mathrm{G})$ \\
\hline $\begin{array}{c}\text { Conventional } \\
2^{\text {nd }} \text {-order loop [3] }\end{array}$ & $20.2 \mathrm{~Hz}$ & $70.2 \mathrm{~Hz}$ & $164.8 \mathrm{~Hz} / \mathrm{s}(3.2 \mathrm{G})$ \\
\hline $\begin{array}{c}\text { Conventional } \\
\text { rd }\end{array}$ & $25.6 \mathrm{~Hz}$ & $95.3 \mathrm{~Hz}$ & $221.5 \mathrm{~Hz} / \mathrm{s}(4.3 \mathrm{G})$ \\
\hline $\begin{array}{c}\text { Conventional } \\
4^{\text {th }} \text {-order loop [3] }\end{array}$ & $16.4 \mathrm{~Hz}$ & $17.8 \mathrm{~Hz}$ & $118.5 \mathrm{~Hz} / \mathrm{s}(2.3 \mathrm{G})$ \\
\hline
\end{tabular}

rier loop does achieve three better acquisition performances: 1) larger frequency ramp range $(515 \mathrm{~Hz} / \mathrm{s}) ; 2)$ wider lock range $(100.2 \mathrm{~Hz})$ and pull-in range $(134.6 \mathrm{~Hz})$; and 3$)$ shorter settling time for the L1 carrier signal. These results demonstrate that our receiver is capable of faster acquisition speed and broader frequency ramp range compared to those of the conventional constant-bandwidth loops in a variety of mobile circumstances.

\section{REFERENCES}

[1] D. Simon and H. El-Sherief, "Fuzzy logic digital phase-locked loop filter design," IEEE Trans. Fuzzy Syst., vol. 3, pp. 211-218, May 1995.

[2] M. S. Braasch and A. J. Van Dierendonck, "GPS receiver architectures and measurements," Proc. IEEE, vol. 87, pp. 48-64, Jan. 1999.

[3] E. D. Kaplan, Understanding GPS: Principles and Applications. London, U.K.: Artech House, 1996.

[4] J. B. Y. Tsui, Fundamentals of Global Positioning System Receivers: A Software Approach. New York: Wiley, 2000.

[5] B. Kosko, Neural Networks and Fuzzy Systems: A Dynamical System Approach to Machine Intelligence. Upper Saddle River, NJ: PrenticeHall, 1992.

[6] W. Zhuang, "Performance analysis of GPS carrier phase observable," IEEE Trans. Aerosp. Electron. Syst., vol. 30, pp. 754-767, Apr. 1996.

[7] J. Lee and B. Kim, "A low-noise fast-lock phase-locked loop with adaptive bandwidth control," IEEE J. Solid-State Circuits, vol. 35, pp. 1137-1145, Aug. 2000.

[8] Y. Tang, Y. Zhou, S. Bibykl, and M. Ismail, "A low-noise fast-settling PLL with extended loop bandwidth enhancement by new adaptation technique," in Proc. 14th Аnпи. IEEE Int. C/SOC Conf., 2001, pp. 93-97. 